\title{
Negative Basis Measurement: Finding the Holy Scale
}

\author{
German Bernhart and Jan-Frederik Mai
}

\begin{abstract}
Investing into a bond and at the same time buying CDS protection on the same bond is known as buying a basis package. Loosely speaking, if the bond pays more than the CDS protection costs, the position has an allegedly risk-free positive payoff known as "negative basis". However, several different mathematical definitions of the negative basis are present in the literature. The present article introduces an innovative measurement, which is demonstrated to fit better into arbitrage pricing theory than existing approaches. This topic is not only interesting for negative basis investors. It also affects derivative pricing in general, since the negative basis might act as a liquidity spread that contributes as a net funding cost to the value of a transaction; see Morini and Parampolini (Risk, 58-63, 2011, [23]).
\end{abstract}

Keywords Negative basis measurement $\cdot$ Bond-CDS basis $\cdot$ Hidden yield

\section{Introduction}

On first glimpse, it is surprising that investing into a bond and buying CDS protection on that underlying bond, henceforth called a basis package, can earn an attractive spread on top of the risk-free rate of return, as it appears to be free of default risk. This excess return over the risk-free rate is informally called negative basis ${ }^{1}$; more formal definitions are given in the main body of this article. [8] has even devoted an entire book to the topic. If, conversely, the cost of CDS protection exceeds the bond earnings, one speaks of a positive basis. In this article, we only speak of negative bases, as fundamentally the concepts of positive and negative basis are simply inverse.

\footnotetext{
${ }^{1}$ Sometimes also called bond-CDS basis.;

G. Bernhart · J.-F. Mai $(\varangle)$

XAIA Investment, Sonnenstraße 19, 80331 München, Germany

e-mail: jan-frederik.mai@xaia.com

G. Bernhart

e-mail: german.bernhart@xaia.com

(C) The Author(s) 2016

K. Glau et al. (eds.), Innovations in Derivatives Markets, Springer Proceedings

in Mathematics \& Statistics 165, DOI 10.1007/978-3-319-33446-2_18
} 
The appropriate measurement of negative basis plays an important role with regard to the cost of funding literature, which has become of paramount interest in the financial industry since the recent liquidity crisis. Generally speaking, this stream of literature reconsiders the pricing of derivatives under the new post-crisis fundamentals regarding funding, liquidity, and credit risk issues. Substantial contributions have been made, among others, by [5, 7, 12, 13, 23, 27, 29]. Loosely speaking, most references agree upon the fact that, at least under certain simplifying assumptions (full, bilateral, and continuous collateralization), derivative contracts can be evaluated in the traditional way, only the involved discount factors have to be adjusted by means of a spread accounting for funding and liquidity charges. In particular, [23] show in a simple, theoretical framework that the negative basis is a spread which plays an essential role in this regard. In order to set these theoretical findings into action in the industry's pricing machinery, it is therefore an essential task to establish viable and reasonable measurements for the negative basis. The present article shows that this topic is not only important but also challenging, and contributes a careful comparison of three different measurement methods. In particular, we point out why the most common measurement approaches (denoted by $(\mathrm{Z})$ and (PE) below) are not recommended, and propose a decent alternative.

In the present article, we take the point of view of a negative basis investor whose goal is to detect interesting negative basis positions and to monitor the evolution of such investments over time. Alternatively, consider a bank which has to evaluate its derivative book. As the aforementioned references show that the required discount factors for the pricing algorithms might have to be adjusted by means of the negative basis, one faces the task of measuring this negative basis appropriately. For the effective implementation of these tasks, it is crucial to come up with a reliable and viable, yet reasonable mathematical definition of what the negative basis actually is. Specific focus is put on simple-to-implement approaches that rely on commonly applied pricing methodologies for bonds and CDS, described in, e.g., [18, 25]. In total, we discuss three different measurements (two traditional and one innovative):

- Difference between Z-spread of the bond and CDS running spread, as presented, e.g., in [8], and defined by Bloomberg on the screen YAS.

- Par-equivalent CDS-methodology, as described in the Appendix of [2], who apply this definition for an empirical study, see also [3].

- A hidden yield approach that assumes the risk-free discounting curve to be a reference interest rate curve shifted by the (initially unknown) negative basis.

Important to note is that, according to all these definitions, a negative basis is assigned to a bond, not to an issuer. This means that two different bonds issued by the same company are allowed to have two different negative bases. This viewpoint stands in glaring contrast to some of the more macro-economic considerations carried out in references cited in the next section. CDS protection typically refers to a whole battery of eligible bonds by a reference issuer, and normally the major driver for CDS spreads is considered to be the issuer's default risk. However, some of the deliverable bonds might trade at diverse yields for reasons other than the issuer's 
default risk-for instance legal issues, liquidity issues, or funding issues, cf. [21] and Sect. 2.

The rest of this article is organized as follows. Section 2 recalls reasons for the existence of negative basis. Section 3 introduces general notations, which are used throughout the remaining sections. Section 4 reviews the traditional methods $(Z)$ and (PE), Sect. 5 discusses the innovative method (HY), and Sect. 6 concludes.

\section{Why Does Negative Basis Exist?}

There are a couple of intuitive explanations for the existence of negative basis, see, e.g., $[1,2,4,6,10,19,24,26,30]$. For the convenience of the reader, we briefly recall some of them in the sequel.

- Liquidity issues: Some bond issues are distributed only among a few investors. If one of these investors has to sell her bonds, for instance due to regulatory requirements or demand for liquidity, supply may exceed demand and thus the price of the bond must drop significantly in order for the bond to be sold. At the same time the CDS price might remain unaffected.

- Funding costs: From a pure credit risk perspective, selling CDS protection economically is the same risk as buying the underlying bond. However, buying a bond requires an initial investment that must be funded, whereas selling CDS protection typically requires much less initial funding (unless the CDS upfront exceeds the bond price). Therefore, in times of high funding costs there is an incentive to sell CDS rather than to buy bonds, which might lead to an increase in supply of CDS protection, making it cheap relative to bond prices.

- Market segmentation: Empirical observations suggest that bond trades sometimes have larger volumes and might be motivated much less by quantitative aspects than CDS trades. Arguing similarly, [6, p. 5, 1.5-7] conjecture that "marketimplied [risk] measures have a stronger impact on the CDS market, while the more easily available rating information affects the bond market more strongly". Such instrument-specific differences might contribute to the existence of negative basis.

- Legal risk: The bond of the negative basis position might bear certain risks that cannot be protected against by means of a CDS. Examples are certain collective action clauses, debt restructuring events, or call rights for the bond issuer. Such "legal gaps" explain parts of the negative basis.

- Counterparty credit risk: A joint default of both the CDS counterparty and the issuer of the bond could lead to a loss for the basis position. ${ }^{2}$ These potential losses imply that CDS protection is not $100 \%$ and consequently might contribute to the negative basis, see, e.g., [5, 22].

- Mark-to-market risk: The negative basis might further increase after one has entered into the position, due to one of the aforementioned reasons. In this case, one

\footnotetext{
${ }^{2}$ However, counterparty credit risk can be reduced significantly by a negative basis investor when the CDS is collateralized, which is the usual case.
} 
loses money due to mark-to-market balancing. In theory, one gets this money back eventually, but it might occur that mark-to-market losses exceed one's personal tolerance level during the bond's lifetime. In this case, one has to exit the position and realize the loss. This risk is especially significant if the negative basis position is levered (which has happened heavily during the financial crisis). Part of the negative basis might be viewed as a risk premium for taking this mark-to-marketrisk.

Basis "arbitrageurs" are investors that try to earn the negative basis by investing into basis packages. This means that they consider the negative basis an adequate compensation for taking the aforementioned risks. In classical arbitrage theory, their appearance improves trading liquidity. Counterintuitively, however, [9] argue that the advent of CDS was detrimental to bond markets and [20] find some evidence that basis arbitrageurs bring new risks into the corporate bond markets.

\section{General Notations}

All definitions to follow rely on the pricing of CDS and a plain vanilla coupon bond according to the most simple mathematical setup we can think of. This is in order to make the article as reader-friendly as possible; furthermore, we think the setup is already rich enough in order to convey the main ideas. The only randomness considered in the present article is the default time of the bond issuer, which is formally defined on a probability space $(\Omega, \mathscr{F}, \mathbb{Q})$, with state space $\Omega, \sigma$-algebra $\mathscr{F}$, and probability measure $\mathbb{Q}$. Expected values with respect to the pricing measure $\mathbb{Q}$ are denoted by $\mathbb{E}$. The default intensity $\lambda($.) of the issuer's default time $\tau$ is assumed to be deterministic, i.e. $\mathbb{Q}(\tau>t)=\exp \left(-\int_{0}^{t} \lambda(s) \mathrm{d} s\right)$. Sometimes the function $\lambda($.$) is$ constant, sometimes piecewise constant, depending on our application. For example, the computation of a so-called Z-spread requires $\lambda\left(\right.$.) to be constant, ${ }^{3}$ whereas the joint consistent pricing of several CDS quotes with different maturities requires $\lambda($.) to be piecewise constant.

Generally speaking, it is our understanding that a negative basis is a measure for the mispricing between CDS and bonds with respect to default risk alone. This explains why considering the default time as the sole stochastic object corresponds to the most minimal modeling approach possible. Besides the non-randomness of the default intensity, the following further simplifying assumptions are taken for granted throughout:

- We ignore recovery risk: Upon default, the bond holder receives the constant proportion $R \in[0,1]$ of her nominal. Default is assumed to instantaneously trigger a credit event of the CDS. The bond is assumed to be a deliverable security in the auction following the CDS trigger event, and the auction process is assumed to yield the same recovery rate $R$. Although this is an unrealistic assumption in

\footnotetext{
${ }^{3}$ See below in Step 3 of Definition 1.
} 
principle (see, e.g., [17]), a negative basis investor can always eliminate recovery risk by delivering his bonds into the auction (physical settlement), in which case he gets compensated by the (nominal-matched) CDS for the nominal loss of the bond. ${ }^{4}$ Consequently, our assumption is not severe for the present purpose.

- We ignore interest rate risk: The discounting curve is deterministic and the discount factors are denoted by $D F(t):=\exp \left(-\int_{0}^{t} r(s) \mathrm{d} s\right)$ with some given deterministic short rate function $r($.$) . All presented negative basis figures are measurements$ relative to the applied short rate function $r($.).

Under these assumptions we introduce the following notations:

- $t_{j}^{(B)}$ denotes the coupon payment dates of the bond.

- The bond's lifetime is denoted by $T$, i.e. $T$ denotes the last coupon payment date, which at the same time is the redemption date. Moreover, the bond is assumed to pay a constant coupon rate $C$ at each coupon payment date.

- $t_{i}^{(C)}$ denotes the payment dates of the considered CDS contracts, which typically are quarterly on the 20th of March, June, September, and December, respectively, according to the terms and conditions of ISDA standard contracts. ${ }^{5}$

- For a CDS with maturity $T$, the (usually standardized) running coupon is denoted by $s(T)$ and the upfront payment to be made at CDS settlement by upf $(T)$.

- The expected discounted value of the sum of all premium payments to be made by the CDS protection buyer (the premium leg) is denoted by ${ }^{6}$

$$
\begin{aligned}
& E D P L(\lambda(.), r(.), s(T), \operatorname{upf}(T), T) \\
& \quad:=\operatorname{upf}(T)+s(T) \sum_{0<t_{i}^{(C)} \leq T}\left(t_{i}^{(C)}-t_{i-1}^{(C)}\right) D F\left(t_{i}^{(C)}\right) \mathbb{Q}\left(\tau>t_{i}^{(C)}\right) \\
& =\operatorname{upf}(T)+s(T) \sum_{0<t_{i}^{(C)} \leq T}\left(t_{i}^{(C)}-t_{i-1}^{(C)}\right) D F\left(t_{i}^{(C)}\right) e^{-\int_{0}^{t_{i}^{(C)}} \lambda(s) \mathrm{d} s} .
\end{aligned}
$$

- The expected discounted value of the sum of all default compensation payments to be made by the CDS protection seller (the default/protection leg) is denoted by

$$
\begin{aligned}
\operatorname{EDDL}(\lambda(.), r(.), R, T): & =(1-R) \mathbb{E}\left[1_{\{\tau \leq T\}} D F(\tau)\right] \\
& =(1-R) \int_{0}^{T} D F(y) \lambda(y) e^{-\int_{0}^{y} \lambda(s) \mathrm{d} s} \mathrm{~d} y .
\end{aligned}
$$

\footnotetext{
${ }^{4}$ Interestingly, a mismatch between bond and CDS recovery is often favorable for the negative basis investor, since the CDS recovery rate tends to be lower than the bond recovery, see, e.g., [14]. Thus, it might make sense for a negative basis investor to opt for cash settlement of the CDS and sell his bonds in the marketplace, speculating on a favorable recovery mismatch.

${ }^{5}$ See http://www2.isda.org/asset-classes/credit-derivatives/.

${ }^{6}$ For the sake of notational convenience we ignore accrued interest upon default, which can, of course, be incorporated easily.
} 
- The model price of the bond is given by

$$
\begin{aligned}
\operatorname{Bond}(\lambda(.), r(.), R, C, T):= & C \sum_{0<t_{j}^{(B)} \leq T}\left(t_{j}^{(B)}-t_{j-1}^{(B)}\right) D F\left(t_{j}^{(B)}\right) \mathbb{Q}\left(\tau>t_{j}^{(B)}\right) \\
& +D F(T) \mathbb{Q}(\tau>T)+R \mathbb{E}\left[1_{\{\tau \leq T\}} D F(\tau)\right] \\
= & C \sum^{(B)}\left(t_{j}^{(B)}-t_{j-1}^{(B)}\right) D F\left(t_{j}^{(B)}\right) e^{-\int_{0}^{t_{j}^{(B)}} \lambda(s) \mathrm{d} s} \\
& 0<t_{j}^{(B)} \leq T \\
+ & D F(T) e^{-\int_{0}^{T} \lambda(s) d s}+R \int_{0}^{T} D F(y) \lambda(y) e^{-\int_{0}^{y} \lambda(s) \mathrm{d} s} \mathrm{~d} y .
\end{aligned}
$$

\section{Traditional Measurements}

\subsection{The Z-Spread Methodology}

The main idea of the Z-spread methodology is to define the negative basis as the difference between (expected) annualized bond earnings and annualized protection costs. This method is described, e.g., in [8]. The negative basis $N B^{(Z)}$ is computed by the following algorithm.

\section{Definition 1 (Negative Basis (Z))}

1. A reference discounting curve, resp. the associated short rate $r($.$) , is chosen and$ used in all subsequent steps, e.g. bootstrapped from quoted prices for interest rate derivatives according to one of the methods described in $[15,16]$.

2. From a term structure of quoted CDS with different maturities, piecewise constant intensities $\lambda($.$) are bootstrapped, as described, e.g., in [25]. For this, a recovery$ assumption is made, i.e. $R$ is model input. ${ }^{7}$

3. Denoting by $B$ the quoted market price of the bond, the bond's $\mathbf{Z}$-spread $z$ is defined as the root of the function ${ }^{8}$

$$
x \mapsto \operatorname{Bond}(x, r(.), 0, C, T)-B,
$$

\footnotetext{
${ }^{7}$ If CDS prices are quoted in running spreads with zero upfronts, then these quotes typically come naturally equipped with a recovery assumption that is required in order to convert the running spreads into actually tradable standardized coupon and upfront payments. However, after this conversion the recovery rate is a free model parameter.

${ }^{8}$ For a reader-friendly explanation of the Z-spread see [28]. In particular, it is useful to observe that $\operatorname{Bond}(x, r(), R, C, T)=.\operatorname{Bond}(0, r()+x, R, C, T$.$) for R=0$, implying that the Z-spread equals a constant default intensity under a zero recovery assumption.
} 
if existent. In words, the Z-spread is the amount by which the reference short rate $r($.) needs to be shifted parallelly in order for the discounted bond cash flows to match the market quote. The root, whenever existing at all, is unique.

4. The (zero-upfront) running CDS spread $s(T)$ for a CDS contract, whose maturity matches the bond's maturity, is defined as

$$
s(T):=\frac{E D D L(\lambda(.), r(.), R, T)}{\operatorname{EDPL}(\lambda(.), r(.), 1,0, T)},
$$

i.e. the fair running spread when no upfront payment is present.

5. $N B^{(Z)}:=z-s(T)$.

Intuitively, the Z-spread $z$ is a measure of the annualized excess return of the bond on top of the "risk-free" rate $r($.$) , whereas s(T)$ is the annualized CDS protection cost. Hence, $N B^{(Z)}$ equals the difference between earnings and costs (expected in case of survival). If the function (1) does not have a root in $(0, \infty)$, this means that the bond is less risky than the default risk intrinsic in the chosen discounting curve $r($.$) . Especially since the liquidity crisis, when the interbank money transfer ran$ dry, significant spreads between discounting curves obtained from overnight rates and LIBOR-based swap rates are observed. Consequently, one could recognize, e.g., German government bonds with a "negative Z-spread" with respect to the interest rate curve $r($.$) , which was obtained from 6-month EURIBOR swap rates. For such$ reasons it has become market standard to extract the "risk-free" discounting curve from overnight rates rather than from LIBOR-based swap rates. Moreover, [19] point out that the difference between bond yields and CDS spreads can depend on whether treasury rates or swap rates are used for discounting. Since negative basis investors are typically trading in the high yield sector, the function (1) normally does have a root in $(0, \infty)$ for several canonical choices of $r($.$) , be it extracted from swap$ rates with overnight tenor, 3-month tenor, or 6-month tenor. But it is important to stress that all presented negative basis measurements are always relative measures depending on the applied interest rate curve $r($.$) .$

The Z-spread methodology has some drawbacks:

- Imprecision: Earnings and costs are not measured accurately, but only approximately. The $Z$-spread is only a rough estimate for the expected annualized earnings, and the zero-upfront running CDS spread is also not really tradable, but only a fictitious quantity. Furthermore, the $Z$-spread is earned on the bond value, whereas the CDS spread is paid on the (bond and) CDS nominal, which may result in a nonsense measurement for bonds trading away from par, see Example 1 below. To this end, [10] proposes to replace the $Z$-spread by an asset swap spread. It is possible to define more accurate measurements of earnings and costs taking into account actual cash flows. However, in the present article we do not elaborate on these fine-tunings, since the "earnings and costs"-perspective in general suffers from the following second difficulty.

- Inaccurate hedge: The measurement assumes that bond and CDS have the same maturity and nominals and furthermore implicitly assumes a survival 
until maturity. Upon a default event the PnL of the position might be considerably different, depending on the timing of the default, see Fig. 1 in Example 1 below. Hence, the assumed CDS hedge cannot really be considered to be default-risk eliminating (it might either profit from or lose on a default event), and consequently the number $N B^{(Z)}$ does not deserve to be called a return figure after elimination of default risk, which the negative basis should be in our opinion.

\subsection{The Par-Equivalent CDS Methodology}

The par-equivalent CDS methodology is described in the Appendix of [2]. A similar idea is also outlined in [8, p. $101 \mathrm{ff}]$ and [3]. The negative basis $N B^{(P E)}$ is computed along the steps of the following algorithm.

\section{Definition 2 (Negative Basis (PE))}

1. A reference discounting curve, resp. the associated short rate $r($.$) , is chosen and$ used in all subsequent steps, e.g. bootstrapped from quoted prices for interest rate derivatives according to one of the methods described in $[15,16]$.

2. From a term structure of CDS contracts on the reference entity, piecewise constant intensities $\lambda($.) are bootstrapped, as described, e.g., in [25]. For this a recovery assumption is made, i.e. $R$ is model input.

3. The (zero-upfront) running CDS spread $s(T)$ for a CDS contract, whose maturity matches the bond's maturity, is defined as

$$
s(T):=\frac{E D D L(\lambda(.), r(.), R, T)}{\operatorname{EDPL}(\lambda(.), r(.), 1,0, T)},
$$

i.e. the fair running spread when no upfront payment is present.

4. Denoting by $B$ the quoted market price of the bond, a shift $\tilde{z}$ is defined as the root of the function

$$
x \mapsto \operatorname{Bond}(\lambda(.)+x, r(.), R, C, T)-B,
$$

if existent. In words, the bond is priced with the default intensities $\lambda($.) that are consistent with CDS quotes, which are then shifted parallelly until the bond's market quote is matched.

5. A second (zero-upfront) running $\operatorname{CDS} \operatorname{spread} \tilde{s}(T)$ for a CDS contract, whose maturity matches the bond's maturity, is defined as

$$
\tilde{s}(T):=\frac{\operatorname{EDDL}(\lambda(.)+\tilde{z}, r(.), R, T)}{\operatorname{EDPL}(\lambda(.)+\tilde{z}, r(.), 1,0, T)}
$$

i.e. the fair spread when no upfront payment is present, but now with the shifted intensity rates $\lambda()+.\tilde{z}$, which are required in order to price the bond correctly.

6. $N B^{(P E)}:=\tilde{s}(T)-s(T)$. 
The main idea of (PE) is to question the default probabilities bootstrapped from the given CDS quotes, and to adjust them in order to match the bond quote. On a high level, this negative basis measurement is based on the difference between default probabilities that are required in order to match the bond price and default probabilities that are required in order to fit the CDS quotes.

The methodology (PE) has some drawbacks:

- No link to arbitrage pricing theory: In our view, there is no convincing economic argument as to why two different survival functions for the same default time should be used. In particular, the method provides no joint pricing model for bond and CDS that explains the negative basis as one of its parameters. The method is "decoupled" from arbitrage pricing theory.

- No link to "earnings and costs"-perspective: Unlike the method $(Z)$, the method $(P E)$ does not have a clear link to an earnings measure above a reference rate, which is what the negative basis is informally thought of.

\section{An Innovative Methodology}

In our opinion, the negative basis should be a spread on top of a reference discounting curve which can be earned without exposure to default risk. This means we question the usual assumption that the applied discounting curve $r($.$) is the appropriate risk-$ free rate to be used, because there is actually a higher rate that can be earned "riskfree" (recalling that default risk is the only risk within our tiny model). This motivates what we call the hidden yield approach. The negative basis $N B^{(H Y)}$ is computed along the steps of the following algorithm.

\section{Definition 3 (Negative Basis $(H Y)$ )}

1. A reference discounting curve, resp. the associated short rate $r($.$) , is chosen and$ used in all subsequent steps, e.g. bootstrapped from quoted prices for interest rate derivatives according to one of the methods described in $[15,16]$.

2. Denote by $\lambda_{x}($.$) the piecewise constant intensity rates that are bootstrapped from$ CDS market quotes, when the assumed discounting curve is $r()+$.$x , as described,$ e.g., in [25]. The recovery rate $R$ is fixed and chosen as model input.

3. The negative basis $N B^{(H Y)}$ is defined as the $\operatorname{root}^{9}$ of the function

$$
x \mapsto \operatorname{Bond}\left(\lambda_{x}(.), r(.)+x, R, C, T\right)-B .
$$

In words, $N B^{(H Y)}$ is precisely the parallel shift of the reference short rate $r($. which allows for a calibration such that the model prices of bond and CDS match the observed market quotes for bond and CDS.

\footnotetext{
${ }^{9}$ Lemma A.1 in the Appendix guarantees that this root typically exists and is unique.
} 
The idea of method (HY) can also be summarized as follows: If the risk-free interest rate curve is assumed to be $r()+.N B^{(H Y)}$, then the market quotes for bond and CDS are arbitrage-free (as we have found a corresponding pricing measure). It allows for the intuitive interpretation of the negative basis as a spread earned on top of a reference discounting rate after elimination of default risk. Abstractly speaking, assuming no transaction costs and availability of CDS protection at all maturities $T>0$ (= perfect market conditions), arbitrage pricing theory suggests the existence of a trading strategy which buys the bond and hedges it via CDS, and which earns ${ }^{10}$ precisely the rate $r()+.N B^{(H Y)}$ until the minimum of default time $\tau$ and bond maturity $T$. Since this way of thinking about $N B^{(H Y)}$ is its distinctive property and highlights its intrinsic coherence with arbitrage pricing theory, the following lemma demonstrates by a heuristic argument how the rate $r()+.N B^{(H Y)}$ can be earned in a risk-free way.

Lemma 1 (The rate $r()+.N B^{(H Y)}$ can be earned without default risk) Assuming perfect market conditions, there exists a (static) portfolio, which is long the bond and invested in several $C D S$, which earns the rate $r()+.N B^{(H Y)}$ until $\min \{\tau, T\}$.

Proof (heuristic) We denote by $\mathbb{Q}$ the probability measure under which $\tau$ has piecewise constant default intensity $\lambda_{N B^{(H Y)}}($.). We discretize the time interval $[0, T]$ into $m$ buckets $0=: t_{0}<t_{1}<\ldots<t_{m}:=T$, but $m$ may be chosen arbitrarily large such that the mesh of the discrete-time grid tends to zero as $m$ tends to infinity. We introduce the following $m+1$ probabilities:

$$
w_{j}^{(m)}:=\mathbb{Q}\left(\tau \in\left(t_{j-1}, t_{j}\right]\right), \quad j=1, \ldots, m, \quad w_{m+1}^{(m)}:=\mathbb{Q}\left(\tau>t_{m}\right) .
$$

Now let $\tau^{(m)}$ denote a random variable with distribution

$$
\begin{aligned}
& \mathbb{Q}\left(\tau^{(m)}=\bar{t}_{j}\right)=w_{j}^{(m)}, \quad \bar{t}_{j}:=\frac{t_{j-1}+t_{j}}{2}, \quad j=1, \ldots, m, \\
& \mathbb{Q}\left(\tau^{(m)}>t\right)=\mathbb{Q}(\tau>t), \quad t \geq t_{m},\left(\text { in particular, } \mathbb{Q}\left(\tau^{(m)}>t_{m}\right)=w_{m+1}^{(m)}\right) .
\end{aligned}
$$

Notice that $\tau^{(m)} \approx \tau$ in distribution, with the approximation improving with increasing $m$. In the sequel, we work with $\tau^{(m)}$, assuming that default during $[0, T]$ can only take place at the possible realizations $\bar{t}_{1}, \ldots, \bar{t}_{m}$ of $\tau^{(m)}$ in $[0, T]$. We now consider a portfolio of $m+1$ instruments, namely the bond and one CDS for each maturity $t_{1}, \ldots, t_{m}$. We assume that the bond nominal is given by $N_{0}$. Furthermore, $N_{i} \in \mathbb{R}$ denotes the nominal of the CDS with maturity $t_{i}$. Negative nominal means that we sell the bond or sell CDS protection. Let's have a look at the following random variables, which are functions of $\tau^{(m)}$ :

\footnotetext{
${ }^{10} \mathrm{By}$ "earning" $r()+.N B^{(H Y)}$ we mean that the internal rate of return of the position is the reference rate $r($.$) plus a spread N B^{(H Y)}$.
} 


$$
\begin{aligned}
V^{(0)}\left(\tau^{(m)}\right):=\left(r(.)+N B^{(H Y)}\right) \text {-discounted value of all cash flows from the bond, } \\
\text { when default takes place at } \tau^{(m)}, \\
V^{(i)}\left(\tau^{(m)}\right):=\left(r(.)+N B^{(H Y)}\right) \text {-discounted value of all cash flows from the CDS with } \\
\text { maturity } t_{i} \text {, when default takes place at } \tau^{(m)}, \quad i=1, \ldots, m .
\end{aligned}
$$

All random variables $V^{(i)}\left(\tau^{(m)}\right)$ take on only $m+1$ possible values, since their value on the event $\left\{\tau^{(m)}>t_{m}\right\}$ does not depend on $\tau^{(m)}$ (as there are no cash flows after $\left.t_{m}\right)$. So without loss of generality we may write $V^{(i)}\left(\tau^{(m)}\right)=V^{(i)}\left(\bar{t}_{m+1}\right)$ for some arbitrary $\bar{t}_{m+1}>t_{m}$ on the event $\left\{\tau^{(m)}>t_{m}\right\}$. Our goal is to show that it is possible to find a non-zero vector $\left(N_{0}, \ldots, N_{m}\right) \in \mathbb{R}^{m+1}$ such that

$$
\underbrace{N_{0} V^{(0)}\left(\tau^{(m)}\right)+\sum_{i=1}^{m} N_{i} V^{(i)}\left(\tau^{(m)}\right)}_{\left(r(.)+N B^{(H Y)}\right) \text {-discounted value of outcome }} \equiv \underbrace{N_{0} B+\sum_{i=1}^{m} N_{i} \operatorname{upf}\left(t_{i}\right)}_{\text {initial investment amount }},
$$

where $B$ denotes the market bond price and upf $\left(t_{i}\right)$ the market upfront of the CDS with maturity $t_{i}$. This mathematical statement intuitively means that the considered portfolio of bond and CDS earns the rate $r()+.N B^{(H Y)}$ until $\min \left\{\tau^{(m)}, T\right\}$ in a riskfree manner, regardless of the actual timing of the default. Now why is this possible? Considering the randomness on the left-hand side of Eq. (2), we actually have $m+1$ equations for the $m+1$ unknowns $N_{0}, N_{1}, \ldots, N_{m}$. Rewriting Eq. (2) in terms of linear algebra, we obtain

$$
\left(\begin{array}{cccc}
V^{(0)}\left(\bar{t}_{1}\right)-B & V^{(1)}\left(\bar{t}_{1}\right)-\operatorname{upf}\left(t_{1}\right) & \ldots & V^{(m)}\left(\bar{t}_{1}\right)-\operatorname{upf}\left(t_{m}\right) \\
\vdots & \ddots & & \vdots \\
\vdots & & \ddots & \vdots \\
V^{(0)}\left(\bar{t}_{m+1}\right)-B & V^{(1)}\left(\bar{t}_{m+1}\right)-\operatorname{upf}\left(t_{1}\right) & \ldots & V^{(m)}\left(\bar{t}_{m+1}\right)-\operatorname{upf}\left(t_{m}\right)
\end{array}\right)\left(\begin{array}{c}
N_{0} \\
N_{1} \\
\vdots \\
N_{m}
\end{array}\right)=\left(\begin{array}{c}
0 \\
0 \\
\vdots \\
0
\end{array}\right) .
$$

In order to prove the existence of a non-trivial solution $\left(N_{0}, \ldots, N_{m}\right)$ to Eq. (3), it suffices to verify that the associated $(m+1) \times(m+1)$-matrix does not have full rank. Now here enters the essential heuristic argument: it follows from the definition of $N B^{(H Y)}$ that

$$
\begin{aligned}
& \sum_{j=1}^{m+1} w_{j}^{(m)} V^{(0)}\left(\bar{t}_{j}\right) \approx B=\sum_{j=1}^{m+1} w_{j}^{(m)} B, \\
& \sum_{j=1}^{m+1} w_{j}^{(m)} V^{(i)}\left(\bar{t}_{j}\right) \approx \operatorname{upf}\left(t_{i}\right)=\sum_{j=1}^{m+1} w_{j}^{(m)} \operatorname{upf}\left(t_{i}\right), \quad i=1, \ldots, m,
\end{aligned}
$$


with the approximations becoming equalities as $m \rightarrow \infty$. In other words, this means that the rows of the equation system (3) are linearly dependent. Consequently, the associated matrix cannot have full rank and the columns must also be linearly dependent, i.e. there exists a non-zero solution $\left(N_{0}, \ldots, N_{m}\right)$ of Eq. (3), and hence (2), as desired. Finally, taking a close look at the structure of the involved cash flows, it is obvious that a solution must satisfy $N_{0} \neq 0$. Without loss of generality we may hence set $N_{0}=1$ (because if $\left(N_{0}, \ldots, N_{m}\right)$ is a solution, so is $\alpha\left(N_{0}, \ldots, N_{m}\right)$ for arbitrary $\alpha \in \mathbb{R}$ ). Concluding, the portfolio we have found is long the bond.

We present an example that demonstrates how different the three presented measurements of negative basis can be in practice. The specifications are inspired by a real-world case.

Example 1 We consider a bond with maturity $T=3.5$ years paying a semi-annual coupon rate of $C=8.25 \%$. It trades far below par value, namely at $B=46.5 \%$. An almost maturity-matched CDS contract is available at an upfront value of $\operatorname{upf}(T)=$ $53 \%$ with a running coupon of $s(T)=5 \%$, payed quarterly. This means a nominalmatched negative basis investment comes at a package price of $46.5+53=99.5 \%$, and pays a coupon rate of $8.25-5=3.25 \%$ until default (however, the bond and CDS coupon payments have different frequencies and payment dates). In the sequel we assume a recovery rate of $R=20 \%$, and the reference rate $r($.$) is bootstrapped$ from 3-month tenor-based interest rate swaps according to the raw interpolation method described in $[15,16]$. Because the bond trades far below par, the measurement $(\mathrm{Z})$ is highly questionable and returns $N B^{(Z)}=-0.42 \%$, which is clearly not an appropriate measurement. As indicated earlier, improved versions of earnings and costs-measurements must be used in order to deal with such extreme situations of highly distressed bonds, but this lies outside the scope of the present article. The par-equivalent CDS methodology returns the measurement $N B^{(P E)}=2.29 \%$, whereas the hidden yield methodology returns the significantly lower number $N B^{(H Y)}=1.18 \%$. While the authors are not aware of a strategy how to monetize the (PE)-measurement $2.29 \%$, Lemma 1 provides a clear interpretation for the (HY)measurement $1.18 \%$ in terms of an internal rate of return that can be earned on top of the risk-free rate, when the negative basis investment is structured as indicated in the proof of Lemma 1.

Now if the described nominal-matched investment seems to earn a rate of $3.25 \%$, which equals a spread of around $1.75 \%$ above the chosen reference rate $r($.$) in$ the present example, why is the measurement $N B^{(H Y)}$ so low? Fig. 1 visualizes the discounted value of the sum over all cash flows from the nominal-matched investment in dependence of the default time. For instance, in case of survival until maturity, this value equals approximately $104 \%$, yielding a return (after discounting) of $5.61 \%$ on the initial investment of $98.39 \%$ (which equals the package price minus accrued CDS coupon, the bond accrued equals zero). Distributed on the 3.5-year investment horizon, this corresponds to a rate of approximately $1.6 \%$ per annum. However, in case of a default just before the first or second bond coupon payment date the 
Fig. 1 Sum over all discounted cash flows arising from the described nominal-matched negative basis investment are depicted, in dependence on the time of default

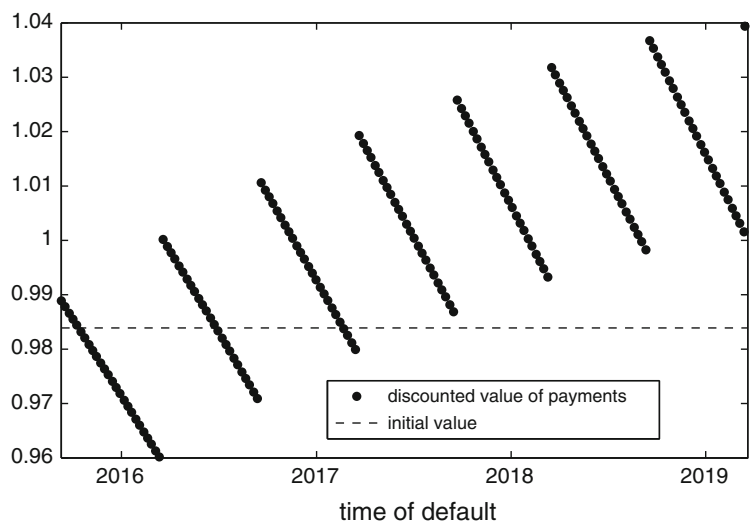

described negative basis investment faces a loss. The additional short-dated CDSprotection required in order to hedge these potential losses decreases the earnings potential of the investment, which is accounted for in the (HY)-methodology, as explained in the proof of Lemma 1.

\section{Conclusion}

We proposed an innovative measurement for the negative basis, denoted $N B^{(H Y)}$. Compared to traditional approaches, it is based on an arbitrage-free pricing model for the simultaneous pricing of the bond and the CDS, which provides a sound economic interpretation. Within a simple model with only default risk being present, the negative basis is perfectly explained as the spread on top of a reference interest rate curve $r($.$) . It was pointed out how the rate r()+.N B^{(H Y)}$ can be earned without exposure to default risk.

Acknowledgements The KPMG Center of Excellence in Risk Management is acknowledged for organizing the conference "Challenges in Derivatives Markets - Fixed Income Modeling, Valuation Adjustments, Risk Management, and Regulation”.

\section{Appendix: The algorithm in Definition 3 is well-defined}

The following technical lemma guarantees that Step 3 in Definition 3 admits a unique solution that can be found efficiently by means of a bisection routine.

Lemma A.1 (Method (HY) is well-defined)

(a) The function $x \mapsto \operatorname{Bond}\left(\lambda_{x}(),. r()+x, R, C, T.\right)$ is continuous. 
(b) The function $x \mapsto \operatorname{Bond}\left(\lambda_{x}(),. r()+x, R, C, T.\right)$ is decreasing on the interval $[-\inf \{r(t): t \geq 0\}, \infty)$.

(c) We have the lower bound Bond $\left(\lambda_{x}(),. r()+x, R, C, T.\right) \geq \frac{R}{1-R} \operatorname{upf}(T)$.

Proof We prove parts (a), (b), and (c) separately.

(a) For fixed $x$, the function $\lambda_{x}($.$) is piecewise constant, so actually we only deal$ with a finite vector of values of the default intensity, depending on $x$. For the remainder of the proof we denote these values by $\left(y_{1}(x), \ldots, y_{m}(x)\right)$. In other words, we observe $m \mathrm{CDS}$ maturities $T_{1}, \ldots, T_{m}$ and the value $y_{k}(x)$ is the level of the default intensity on the piece $\left(T_{k-1}, T_{k}\right]$, for $k=1, \ldots, m$, with $T_{0}:=0$. Obviously, the bond price then equals a concatenation of continuous functions if each $y_{k}(x)$ is continuous in $x$. However, this is guaranteed by the implicit function theorem since $y_{k}(x)$ is defined as the implicit function yielding the root of a smooth function. Concluding, continuity of the bond price is clear.

(b) In order to see that the bond price is decreasing in $x$, we first re-write it as

$$
\begin{aligned}
\operatorname{Bond}\left(\lambda_{x}(.), r(.)+x, R, C, T\right)= & e^{-\int_{0}^{T} \lambda_{x}(s)+r(s)+x \mathrm{~d} s} \\
& +C \sum_{0<t_{j}^{(B)} \leq T}\left(t_{j}^{(B)}-t_{j-1}^{(B)}\right) e^{-\int_{0}^{t_{j}^{(B)}} \lambda_{x}(s)+r(s)+x \mathrm{~d} s} \\
& +\frac{R}{1-R} \operatorname{EDPL}\left(\lambda_{x}(.), r(.)+x, \operatorname{upf}(T), T\right),
\end{aligned}
$$

where we have used $E D P L=E D D L$ from the CDS boostrap. This shows that it suffices to check that the function

$$
x \mapsto \lambda_{x}(t)+x
$$

is increasing for each fixed $t$, because all summands in the above bond formula are then obviously decreasing.

We proceed with an auxiliary observation. If $\tau_{1}$ and $\tau_{2}$ are two positive random variables with distribution functions $F_{1}$ and $F_{2}$, satisfying $F_{1} \geq F_{2}$ pointwise on an interval $(T, \infty)$ and $F_{1} \equiv F_{2}$ on $[0, T]$, then $\mathbb{E}\left[g\left(\tau_{1}\right)\right] \geq \mathbb{E}\left[g\left(\tau_{2}\right)\right]$ for any bounded function $g:(0, \infty) \rightarrow[0, K]$, which is non-increasing on $(T, \infty)$. To verify this, ${ }^{11}$ define the non-decreasing function $h:=-g$ and use integration by parts:

\footnotetext{
${ }^{11}$ One says that $\tau_{1}$ is less than $\tau_{2}$ in the usual stochastic order, and the following computation is standard in the respective theory.
} 


$$
\begin{aligned}
\mathbb{E}\left[g\left(\tau_{1}\right)\right] & =-\int h \mathrm{~d} F_{1}=-\int_{(0, T]} h \mathrm{~d} F_{1}-\int_{(T, \infty)} h \mathrm{~d} F_{1} \\
& =-\int_{(0, T]} h \mathrm{~d} F_{2}-(h(\infty) \underbrace{F_{1}(\infty)}_{=1=F_{2}(\infty)}-h(T) \underbrace{F_{1}(T)}_{=F_{2}(T)}-\int_{(T, \infty)} F_{1} \mathrm{~d} h) \\
& \geq-\int_{(0, T]} h \mathrm{~d} F_{2}-\left(h(\infty) F_{2}(\infty)-h(T) F_{2}(T)-\int_{(T, \infty)} F_{2} \mathrm{~d} h\right) \\
& =-\int_{(0, T]} h \mathrm{~d} F_{2}-\int_{(T, \infty)} h \mathrm{~d} F_{2}=-\int h \mathrm{~d} F_{2}=\mathbb{E}\left[g\left(\tau_{2}\right)\right] .
\end{aligned}
$$

Now we proceed inductively over $k=1, \ldots, m$ by showing that $x \mapsto \lambda_{x}(t)+x$ is non-decreasing for all fixed $t \in\left(T_{k-1}, T_{k}\right]$, i.e. that $x \mapsto y_{k}(x)+x$ is nondecreasing. We start the induction for $k=1$. To this end, recall that $y_{1}(x)$ is the unique root of the equation

$$
E D P L\left(y_{1}(x), r(.)+x, s\left(T_{1}\right), \operatorname{upf}\left(T_{1}\right), T_{1}\right)=E D D L\left(y_{1}(x), r(.)+x, R, T_{1}\right) .
$$

For the sake of a more compact notation we denote the left-hand side of the last equation by $\operatorname{LHS}\left(x, y_{1}(x)\right)$ and the right-hand side by $\operatorname{RHS}\left(x, y_{1}(x)\right)$. Furthermore, we denote the value of both sides by $V(x):=\operatorname{LHS}\left(x, y_{1}(x)\right)=$ $R H S\left(x, y_{1}(x)\right)$. Since all the summands of LHS depend on the function $x \mapsto$ $x+y_{1}(x)$ in a monotonic way, it is obvious that $V(x)$ is non-increasing in $x$ if and only if the function $x \mapsto x+y_{1}(x)$ is non-decreasing. Hence, it suffices to prove that $V(x)$ is non-increasing in $x$. To this end, we (obviously) observe with $\varepsilon>0$ that

$$
\begin{aligned}
& \operatorname{LHS}\left(x+\varepsilon, y_{1}(x)\right) \leq \operatorname{LHS}\left(x, y_{1}(x)\right)=V(x), \\
& \operatorname{RHS}\left(x+\varepsilon, y_{1}(x)\right) \leq \operatorname{RHS}\left(x, y_{1}(x)\right)=V(x) .
\end{aligned}
$$

Furthermore, the function $y \mapsto L H S(x+\varepsilon, y)$ is obviously strictly decreasing. Concerning the right-hand side, we denote by $\mathbb{E}_{y}[f(\tau)]$ the expectation over $f(\tau)$ when the default time $\tau$ has an exponential distribution with parameter $y$. The function

$$
y \mapsto R H S(x+\varepsilon, y)=(1-R) \mathbb{E}_{y}\left[e^{-\int_{0}^{\tau} r(s)+x+\varepsilon \mathrm{d} s} 1_{\left\{\tau \leq T_{1}\right\}}\right]
$$

is non-decreasing on the claimed interval by the auxiliary observation we have derived above (increasing $y$ corresponds to increasing the distribution function of the default time $\tau$ pointwise ${ }^{12}$ ). We now distinguish two cases:

\footnotetext{
${ }^{12}$ Here, we have used that the function $\tau \mapsto \exp \left(-\int_{0}^{\tau} r(s)+x+\varepsilon \mathrm{d} s\right) 1_{\left\{\tau \leq T_{1}\right\}}$ is non-increasing if $x \geq-\inf \{r(t): t \geq 0\}$.
} 
(i) $\operatorname{LHS}\left(x+\varepsilon, y_{1}(x)\right) \leq \operatorname{RHS}\left(x+\varepsilon, y_{1}(x)\right)$ :

In this case $y_{1}(x+\varepsilon) \leq y_{1}(x)$, because otherwise we would observe the following contradiction:

$$
\begin{aligned}
\operatorname{LHS}\left(x+\varepsilon, y_{1}(x+\varepsilon)\right) & <\operatorname{LHS}\left(x+\varepsilon, y_{1}(x)\right) \leq \operatorname{RHS}\left(x+\varepsilon, y_{1}(x)\right) \\
& \leq \operatorname{RHS}\left(x+\varepsilon, y_{1}(x+\varepsilon)\right) .
\end{aligned}
$$

This implies that

$$
V(x+\varepsilon)=R H S\left(x+\varepsilon, y_{1}(x+\varepsilon)\right) \leq R H S\left(x+\varepsilon, y_{1}(x)\right) \stackrel{(5)}{\leq} V(x) .
$$

(ii) $\operatorname{LHS}\left(x+\varepsilon, y_{1}(x)\right)>\operatorname{RHS}\left(x+\varepsilon, y_{1}(x)\right)$ :

In this case $y_{1}(x+\varepsilon) \geq y_{1}(x)$, because otherwise we would observe the following contradiction:

$$
\begin{aligned}
R H S\left(x+\varepsilon, y_{1}(x+\varepsilon)\right) & \leq \operatorname{RHS}\left(x+\varepsilon, y_{1}(x)\right)<\operatorname{LHS}\left(x+\varepsilon, y_{1}(x)\right) \\
& \leq \operatorname{LHS}\left(x+\varepsilon, y_{1}(x+\varepsilon)\right) .
\end{aligned}
$$

This implies that

$$
V(x+\varepsilon)=\operatorname{LHS}\left(x+\varepsilon, y_{1}(x+\varepsilon)\right) \leq \operatorname{LHS}\left(x+\varepsilon, y_{1}(x)\right) \stackrel{(4)}{\leq} V(x) .
$$

Concluding, $V(x)$ is non-increasing in $x$ and the induction start is finished.

We proceed with the induction step, assuming that we already know that $x+\lambda_{x}(t)$ is non-decreasing in $x$ for each fixed $t \leq T_{k-1}$. To this end, recall that $y_{k}(x)$ is the unique root of the equation

$$
\operatorname{EDPL}\left(\lambda_{x}(.), r(.)+x, s\left(T_{k}\right), \operatorname{upf}\left(T_{k}\right), T_{k}\right)=E D D L\left(\lambda_{x}(.), r(.)+x, R, T_{k}\right),
$$

where $y_{k}(x)$ enters the equation as the function value of $\lambda_{x}($.$) on the interval$ $\left(T_{k-1}, T_{k}\right]$. The left-hand side of the last equation can be rewritten as follows, using the standard market convention of standardized CDS strike rates $s\left(T_{k-1}\right)=$ $s\left(T_{k}\right)=: s$ :

$$
\begin{aligned}
& \operatorname{EDPL}\left(\lambda_{x}(.), r(.)+x, s, \operatorname{upf}\left(T_{k}\right), T_{k}\right) \\
& =\operatorname{EDPL}\left(\lambda_{x}(.), r(.)+x, s, \operatorname{upf}\left(T_{k-1}\right), T_{k-1}\right)+\operatorname{upf}\left(T_{k}\right)-\operatorname{upf}\left(T_{k-1}\right) \\
& \quad+s \sum_{T_{k-1}<t_{i}^{(C)} \leq T_{k}}\left(t_{i}^{(C)}-t_{i-1}^{(C)}\right) e^{-\int_{0}^{t_{i}^{(C)}} \lambda_{x}(s)+r(s)+x \mathrm{~d} s} .
\end{aligned}
$$

Similarly, the right-hand side can be rewritten as follows:

$$
\begin{aligned}
\operatorname{EDDL}\left(\lambda_{x}(.), r(.)+x, R, T_{k}\right)= & \operatorname{EDDL}\left(\lambda_{x}(.), r(.)+x, R, T_{k-1}\right) \\
& +(1-R) y_{k}(x) \int_{T_{k-1}}^{T_{k}} e^{-\int_{0}^{t} r(s)+x+\lambda_{x}(s) \mathrm{d} s} \mathrm{~d} t .
\end{aligned}
$$


Since the values $\left(y_{1}(x), \ldots, y_{k-1}(x)\right)$ have been determined before, we may subtract the $E D D L$ and $E D P L$ with maturity $T_{k-1}$ on both sides of the defining equation for $y_{k}(x)$, simplifying the latter to

$$
\begin{aligned}
& \operatorname{upf}\left(T_{k}\right)-\operatorname{upf}\left(T_{k-1}\right)+s \sum_{T_{k-1}<t_{i}^{(C)} \leq T_{k}}\left(t_{i}^{(C)}-t_{i-1}^{(C)}\right) e^{-\int_{0}^{t_{i}} \lambda_{x}(s)+r(s)+x \mathrm{~d} s} \\
& =(1-R) y_{k}(x) \int_{T_{k-1}}^{T_{k}} e^{-\int_{0}^{t} r(s)+x+\lambda_{x}(s) \mathrm{d} s} \mathrm{~d} t .
\end{aligned}
$$

Again, we denote the left-hand side of the last equation by $\operatorname{LHS}\left(x, y_{1}(x), \ldots\right.$, $\left.y_{k}(x)\right)$, and the right-hand side is denoted $\operatorname{RHS}\left(x, y_{1}(x), \ldots, y_{k}(x)\right)$. Furthermore, we denote the value of both sides by

$$
V(x):=\operatorname{LHS}\left(x, y_{1}(x), \ldots, y_{k}(x)\right)=\operatorname{RHS}\left(x, y_{1}(x), \ldots, y_{k}(x)\right) .
$$

By induction hypothesis, the function $x \mapsto x+\lambda_{x}(t)$ is non-decreasing for each $t \leq T_{k-1}$. With $\varepsilon>0$ this obviously implies that

$$
\begin{gathered}
\operatorname{LHS}\left(x+\varepsilon, y_{1}(x+\varepsilon), \ldots, y_{k-1}(x+\varepsilon), y_{k}(x)\right) \\
\leq \operatorname{LHS}\left(x, y_{1}(x), \ldots, y_{k}(x)\right)=V(x), \\
R H S\left(x+\varepsilon, y_{1}(x+\varepsilon), \ldots, y_{k-1}(x+\varepsilon), y_{k}(x)\right) \\
\leq \operatorname{RHS}\left(x, y_{1}(x), \ldots, y_{k}(x)\right)=V(x) .
\end{gathered}
$$

Also, the function $y \mapsto \operatorname{LHS}\left(x+\varepsilon, y_{1}(x+\varepsilon), \ldots, y_{k-1}(x+\varepsilon), y\right)$ is obviously non-increasing, whereas the function $y \mapsto R H S\left(x+\varepsilon, y_{1}(x+\varepsilon), \ldots\right.$, $\left.y_{k-1}(x+\varepsilon), y\right)$ is non-decreasing by a similar argument as in the induction start, namely: the right-hand side has the form ${ }^{13}$

$$
\begin{aligned}
& R H S\left(x+\varepsilon, y_{1}(x+\varepsilon), \ldots, y_{k-1}(x+\varepsilon), y\right) \\
& \quad=(1-R) \mathbb{E}_{y}\left[e^{-\int_{0}^{\tau} r(s)+x+\varepsilon \mathrm{d} s} 1_{\left\{\tau \in\left(T_{k-1}, T_{k}\right]\right\}}\right],
\end{aligned}
$$

which is non-decreasing in $y$. Why? Because an increase of $y$ increases the distribution function of $\tau$ pointwise on $\left[T_{k-1}, \infty\right)$ but leaves it unchanged on $\left[0, T_{k-1}\right]$, and the function $\tau \mapsto \exp \left(-\int_{0}^{\tau} r(s)+x \mathrm{~d} s\right) 1_{\left\{\tau \in\left(T_{k-1}, T_{k}\right]\right\}}$ is clearly non-increasing on $\left(T_{k-1}, \infty\right)$ (so that our auxiliary observation above applies). Like in the induction start, showing that $x \mapsto x+y_{k}(x)$ is non-decreasing in $x$ is equivalent to showing that $V(x)$ is non-increasing in $x$. The remaining proof is now completely analogous to the induction start (this is an exercise we leave to the reader).

\footnotetext{
${ }^{13}$ Similar as in the induction start, we denote by $\mathbb{E}_{y}[f(\tau)]$ the expectation over $f(\tau)$ when the default time has piecewise constant intensity with the level $y$ on the piece $\left(T_{k-1}, T_{k}\right]$.
} 
(c) Denoting by $\mathbb{Q}_{x}$ the probability measure in dependence of the default intensities $\lambda_{x}($.$) , we have$

$$
\begin{aligned}
\operatorname{Bond}\left(\lambda_{x}(.), r(.)+x, R, C, T\right):= & C \sum_{0<t_{j}^{(B)} \leq T} D F\left(t_{j}^{(B)}\right)\left(t_{j}^{(B)}-t_{j-1}^{(B)}\right) \mathbb{Q}_{x}\left(\tau>t_{j}^{(B)}\right) \\
& +D F(T) \mathbb{Q}_{x}(\tau>T)+R \mathbb{E}_{x}\left[1_{\{\tau \leq T\}} D F(\tau)\right] .
\end{aligned}
$$

We know from the consistent CDS pricing that the appearing expectation can be replaced by the premium leg of the CDS, which allows to be estimated by the upfront, i.e.

$$
\begin{aligned}
\mathbb{E}_{x}\left[1_{\{\tau \leq T\}} D F(\tau)\right] & =\frac{R}{1-R} \operatorname{EDPL}\left(\lambda_{x}(.), r(.)+x, s(T), \operatorname{upf}(T), T\right) \\
& \geq \frac{R}{1-R} \operatorname{upf}(T),
\end{aligned}
$$

which in turn implies the claim.

Open Access This chapter is distributed under the terms of the Creative Commons Attribution 4.0 International License (http://creativecommons.org/licenses/by/4.0/), which permits use, duplication, adaptation, distribution and reproduction in any medium or format, as long as you give appropriate credit to the original author(s) and the source, a link is provided to the Creative Commons license and any changes made are indicated.

The images or other third party material in this chapter are included in the work's Creative Commons license, unless indicated otherwise in the credit line; if such material is not included in the work's Creative Commons license and the respective action is not permitted by statutory regulation, users will need to obtain permission from the license holder to duplicate, adapt or reproduce the material.

\section{References}

1. Andritzky, J., Singh, M.: The pricing of credit default swaps during distress. IMF Working Paper 06/254 (2006)

2. Bai, J., Collin-Dufresne, P.: The determinants of the CDS-Bond basis during the financial crises of 2007-2009. Working Paper (2011)

3. Beinstein, E., Scott, A., Graves, B., Sbityakov, A., Le, K., Goulden, J., Muench, D., Doctor, S., Granger, A., Saltuk, Y., Allen, P.: Credit Derivatives Handbook. J.P. Morgan Corporate Quantitative Research (2006)

4. Blanco, R., Brennan, S., Marsh, I.W.: An empirical analysis of the dynamic relation between investment-grade bonds and credit default swaps. J. Financ. 60(5), 2255-2281 (2005)

5. Brigo, D., Capponi, A., Pallavicini, A.: Arbitrage-free bilateral counterparty risk valuation under collateralization and application to Credit Default Swaps. Math. Financ. 24(1), 125-146 (2014)

6. Bühler, W., Trapp, M.: Explaining the bond-CDS basis-the role of credit risk and liquidity. In: Risikomanagement und kapitalmarktorientierte Finanzierung: Festschrift für Bernd Rudolph zum 65. Geburtstag, Knapp-Verlag, Frankfurt a. Main, pp. 375-397 (2009)

7. Burgard, C., Kjaer, M.: In the balance. Risk pp. 72-75 (2011) 
8. Choudhry, M.: The Credit Default Swap Basis. Bloomberg Press, New York (2006)

9. Das, S., Kalimipalli, M.: Did CDS trading improve the market for corporate bonds? J. Financ. Econ. 111(2), 495-525 (2014)

10. De Wit, J.: Exploring the CDS-Bond basis. National Bank of Belgium working paper No. 104 (2006)

11. Doctor, S., White, D., Elizalde, A., Goulden, J., Toublan, D.D.: Differential discounting for CDS: J.P. Morgan Europe Credit Research (2012)

12. Fries, C.: Discounting Revisited: Valuation Under Funding, Counterparty Risk and Collateralization. Working paper (2010), available at SSRN: http://ssrn.com/abstract=1609587

13. Fujii, M., Shimada, Y., Takahashi, A.: Collateral Posting and Choice of Collateral Currency. CIRJE Discussion Papers (2010)

14. Gupta, S., Sundaram, R.K.: Mispricing and arbitrage in CDS auctions. J. Deriv. 22(4), 79-91 (2015)

15. Hagan, P.S., West, G.: Interpolation methods for curve construction. Appl. Math. Financ. 13(2), 89-129 (2006)

16. Hagan, P.S., West, G.: Methods for constructing a yield curve. Wilmott magazine pp.70-81 (2008)

17. Höcht, S., Kunze, M., Scherer, M.: Implied recovery rates-auction and models. In: Innovations in Quantitative Risk Management. Springer, Berlin pp. 147-162 (2015)

18. Hull, J., White, A.: Valuing credit default swaps I: no counterparty default risk. J. Deriv. 8(1), 29-40 (2000)

19. Hull, J., Predescu, M., White, A.: The relationship between credit default swap spreads, bond yields, and credit rating announcements. J. Bank. Financ. 28, 2789-2811 (2004)

20. Li, H., Zhang, W., Kim, G.H.: The CDS-Bond basis and the cross section of corporate bond returns. Working paper (2011)

21. Longstaff, F.A., Neis, E., Mithal, S.: Corporate yield spreads: default risk or liquidity? New evidence from the credit-default swap market. J. Financ. 60(5), 2213-2253 (2005)

22. Mai, J.-F., Scherer, M.: Simulating from the copula that generates the maximal probability for a joint default under given (inhomogeneous) marginals. In: Melas, V.B. et al.: Topics in Statistical Simulation: Springer Proceedings in Mathematics and Statistics, vol. 114, pp. 333341, Springer, Heidelberg (2014)

23. Morini, M., Prampolini, A.: Risky funding with counterparty and liquidity charges. Risk pp. 58-63 (2011)

24. O'Kane, D.: The link between Eurozone sovereign debt and CDS prices. EDHEC-Risk Institute Working Paper (2012)

25. O'Kane, D., Turnbull, S.: Valuation of credit default swaps. Fixed Income Quantitative Research Lehman Brothers (2003)

26. Palladini, G., Portes, R.: Sovereign CDS and bond pricing dynamics in the Euro-area. Centre for Economic Policy Research Discussion Paper No. 8651 (2011)

27. Pallavicini, A., Perini, D., Brigo, D.: Funding Valuation Adjustment: A Consistent Framework Including CVA, DVA, Collateral, Netting Rules and Re-Hypothecation. Working paper (2011), available at SSRN: http://ssrn.com/abstract=1969114

28. Pedersen, C.M.: Explaining the Lehman Brothers option adjusted spread of a corporate bond. Fixed Income Quantitative Credit Research, Lehman Brothers (2006)

29. Piterbarg, V.: Funding beyond discounting: collateral agreements and derivatives pricing. Risk, pp. 97-102 (2010)

30. Zhu, H.: An empirical comparison of credit spreads between the bond market and the credit default swap market. BIS Working Paper No. 160 (2004) 